\title{
A decomposition theorem for positive maps, and the projection onto a spin factor
}

\author{
Erling Størmer
}

08-13-2013

It is shown that each positive map between matrix algebras is the sum of a maximal decomposable map and an atomic map which is both optimal and co-optimal. The result is studied in detail for the projection onto a spin factor.

\section{Introduction}

The structure of positive maps between $C^{*}$-algebras, even in the finite dimensional case, is still poorly understood. The only maps which are well understood are the decomposable ones, which are sums of completely positive and co-positive maps, hence in the finite dimensional case, are sums of maps of the form $A d v$ and $t \circ A d v$, where $t$ is the transpose map, and $A d v$ the map $x \rightarrow v^{*} x v$. In the present paper we shall shed some light on the structure of positive maps by showing that they are the sum of a maximal decomposable map and an atomic map, which is bi-optimal, i.e. it majorizes neither a non-zero completely positive map nor a co-positive map.

In order to obtain a deeper understanding of this decomposition we study it in detail in Section 2 for the trace invariant positive projection of the full matrix algebra $M_{2^{n}}$ onto a spin factor inside it. We shall obtain explicit formulas for the decomposable map and the bi-optimal map in the decomposition when the spin factor is irreducible and contained in the $2^{n-1} \times 2^{n-1}$ matrices over the quaternions.

For the readers convenience we recall the main definitions concerning positive maps, see also 8 . We let $A$ be a finite dimensional $C^{*}$-algebra and $B(H)$ the bounded operators on a finite dimensional Hilbert space $H$.

Let $\phi: A \rightarrow B(H)$ be a linear map. $\phi$ is positive, written $\phi \geq 0$ or $0 \leq \phi$ if it carries positive operators to positive operators. If $\psi$ is another positive map, $\psi$ majorizes $\phi$, written $\psi \geq \phi$ if $\psi-\phi \geq 0$. $\phi$ is $k$-positive if $\iota_{k} \otimes \phi: M_{k} \otimes A \rightarrow$ $M_{k} \otimes B(H)$ is positive, where $\iota_{k}$ is the identity map on the $k \times k$ matrices $M_{k}$. $\phi$ is completely positive if $\phi$ is $\mathrm{k}$-positive for all $\mathrm{k}$. Let $t$ denote the transpose map on $B(H)$ with respect to some fixed orthonormal basis. Then $\phi$ is $k$-copositive, (resp. co-positive) if $t \circ \phi$ is k-positive (resp. completely positive). $\phi$ is $k$-decomposable (resp. decomposable) if $\phi$ is the sum of a k-positive and a k-copositive map (resp. completely positive and a co-positive map). $\phi$ is atomic if 
$\phi$ is not 2-decomposable. $\phi$ is extremal or just extreme, if $\phi \geq \psi$ for a positive map $\psi$ implies $\psi=\lambda \phi$ for some nonnegative number $\lambda$. $\phi$ is optimal (resp. co-optimal) if $\phi \geq \psi$ for $\psi$ completely positive (reps. co-positive) implies $\psi=0$. Combining the last two concepts we introduce the following definition, which has also been introduced by Ha and Kye [2].

Definition $1 \phi$ is bi-optimal if $\phi$ is both optimal and co-optimal.

The author is grateful to E. Alfsen for many helpful discussions on spin factors.

\section{The decomposition theorem}

Let $K$ and $H$ be finite dimensional Hilbert spaces. In [5], Theorem 3.4 Marciniak showed the surprising result that if $\phi$ is a 2-positive map (resps. 2-co-positive) which is extremal, then $\phi$ is completely positive (reps. co-positive). His proof, see also 8 , Theorem 3.3.7, contained more information, namely the following result.

Lemma 2 Let $\phi$ be a non-zero 2-positive map of $B(K)$ into $B(H)$. Then there exists a non-zero completely positive map $\psi: B(K) \rightarrow B(H)$ such that $\phi \geq \psi$.

A slight extension of the above lemma yields the following.

Proposition 3 Let $A$ be a finite dimensional $C^{*}$-algebra and $\phi: A \rightarrow B(H)$ a non-zero 2-decomposable map. Then there exists a non-zero decomposable map $\psi: A \rightarrow B(H)$ such that $\phi \geq \psi$.

Proof. We first consider the case when $A=B(K)$. Since $\phi$ is 2-decomposable there exist a 2-positive map $\phi_{1}$ and a 2-co-positive map $\phi_{2}$ such that $\phi=\phi_{1}+\phi_{2}$. By Lemma 2 there is a completely positive map $\psi_{1}$, non-zero if $\phi_{1}$ is non-zero, such the $\phi_{1} \geq \psi_{1}$. Applying Lemma 2 to $t \circ \phi_{2}$ we find a co-positive map $\psi_{2} \leq \phi_{2}$. Thus $\phi \geq \psi_{1}+\psi_{2}$, proving the proposition when $A=B(K)$.

In the general case let $e_{1}, \ldots, e_{m}$ be the minimal central projections in $A$, so $A=\bigoplus_{i}{ }^{m} A e_{i}$. Then each $A e_{i}$ is isomorphic to some $B(K)$, and $\phi_{\mid A e_{i}}$ is 2decomposable. By the first part $\phi_{\mid A e_{i}} \geq \alpha_{i}+\beta_{i}$ with $\alpha_{i}$ completely positive and $\beta_{i}$ co-positive. Let $\alpha=\sum \alpha_{i}$ and $\beta=\sum \beta_{i}$. Then $\alpha$ is completely positive and $\beta$ co-positive, hence $\alpha+\beta$ is a decomposable map majorized by $\phi$, completing the proof of the proposition.

Corollary 4 Each bi-optimal map of a finite dimensional $C^{*}$-algebra into $B(H)$ is atomic.

Proof. By definition a map $\phi$ is atomic if it is not 2-decomposable. By definition of being bi-optimal such a map $\phi$ cannot majorize a decomposable map, hence by Proposition $3 \phi$ cannot be 2 -decomposable, completing the proof. 
Since completely positive maps are sums of maps of the form $A d v$, and each co-positive map a sum of maps $t \circ A d v$, our next result reduces much of the study of positive maps to that of bi-optimal maps. If $\phi: A \rightarrow B(H)$ is positive, $A$ a $C^{*}$-algebra, we say a decomposable map $\alpha: A \rightarrow B(H), \alpha \leq \phi$ is a maximal decomposable map majorized by $\phi$ if there is no decomposable map $\psi: A \rightarrow B(H)$ such that $\psi \neq \alpha$, and $\alpha \leq \psi \leq \phi$.

Theorem 5 Let $A$ be a finite dimensional $C^{*}$-algebra and $H$ a finite dimensional Hilbert space. Let $\phi: A \rightarrow B(H)$ be a positive map. Then there are a maximal decomposable map $\alpha: A \rightarrow B(H)$ majorized by $\phi$ and a bi-optimal, hence atomic, map $\beta: A \rightarrow B(H)$ such that $\phi=\alpha+\beta$.

Proof. We first assume $A=B(K)$ for a finite dimensional Hilbert space $K$. Let

$$
C=\{\psi: B(K) \rightarrow B(H): \psi \text { decomposable, } \psi \leq \phi\} .
$$

Then $C$ is bounded and norm closed, hence is compact in the norm topology, as $K$ and $H$ are finite dimensional. Furthermore $C$ is an ordered set with the usual ordering on positive maps. We show $C$ has a maximal element. For this let $X=\left\{\phi_{v} \in C: v \in F\right\}$ be a totally ordered set with $\phi_{v} \leq \phi_{v^{\prime}}$ if $v \leq v^{\prime}$ in $F$. For each $v \in F$ let $X_{v}=\left\{\phi_{v^{\prime}} \in X: v \leq v^{\prime}\right\}$. Then $X_{v}$ is closed, and $X_{v} \supset X_{v^{\prime}}$ if $v \leq v^{\prime}$. Since $X$ is totally ordered it follows that the sets $X_{v}$ with $v \in F$ have the finite intersection property. Thus the intersection $\bigcap_{v \in F} X_{v} \neq \emptyset$, hence a map $\psi \in \bigcap X_{v}$ is an upper bound for $X$. By Zorn's lemma $C$ has a maximal element $\alpha$. Since $C$ is closed, $\alpha$ is decomposable, $\alpha \leq \phi$, and there is no decomposeosable map $\psi: B(K) \rightarrow B(H)$ different from $\alpha$ such that $\alpha \leq \psi \leq \phi$. Thus $\alpha$ is maximal decomposable map majorized by $\phi$.

Let $\beta=\phi-\alpha$. Then $\beta$ is bi-optimal, for if $\gamma \leq \beta, \gamma \neq 0$ and decomposable, then $\alpha+\gamma$ is decomposable, and $\alpha+\gamma \leq \alpha+\beta=\phi$, contradicting maximality of $\alpha$. Thus $\gamma=0$, and $\beta$ is bi-optimal.

In the general case we imitate the proof of Proposition 3 and write $A$ as $A=$ $\bigoplus A e_{i}$ where the $e_{i}$ are minimal central projections in $A$, so $A e_{i}$ is isomorphic to some $B(K)$, and we apply the first part of the proof to each $A e_{i}$ in the same way as we did in the proof of Proposition 3. The proof is complete.

If we do not require $\alpha$ in the theorem to be maximal decomposable we can have different decompositions. For example, if $\phi$ is a bi-optimal map, and $T r$ is the trace on $B(K)$, then the map $\psi(x)=\phi(1) \operatorname{Tr}(x)+\phi(x)$ is super-positive, hence in particular completely positive, see [8], Theorem 7.5.4. But $\psi$ has a decomposition $\psi=\alpha+\beta$, where $\alpha=\phi(1) \operatorname{Tr}$ is completely positive, and $\beta=\phi$ is bi-optimal.

Corollary 6 With assumptions as in Theorem 5, if $\phi$ is extreme, then $\phi$ is either of the form Adv, $t \circ A d v$ or $\phi$ is bi-optimal, so atomic.

If we in the proof of Theorem 5 replace decomposable map by completely positive map and bi-optimal by optimal and define maximal completely map majorized by $\phi$ in analogy with the definition for decomposable maps, we obtain the following result. 
Theorem 7 Let $A$ be a finite dimensional $C^{*}$-algebra and $H$ a finite dimensional Hilbert space. Let $\phi: A \rightarrow B(H)$ be a positive map. Then there are a maximal completely positive map $\alpha: A \rightarrow B(H)$ majorized by $\phi$ and an optimal map $\beta: A \rightarrow B(H)$ such that $\phi=\alpha+\beta$.

\section{Spin factors}

In the present section we illustrate the decomposition theorems, Theorem 5 and Theorem 7 , by the projection of $B(H)$ onto a spin factor. Following [3] we recall that a spin system in $B(H)$ is a set of symmetries, i.e. self-adjoint unitaries $s_{1}, \ldots, s_{m}$ satisfying the anti-commutation relations $s_{i} s_{j}+s_{j} s_{i}=0$ for $i \neq j$. Let

$$
\sigma_{1}=\left(\begin{array}{cc}
1 & 0 \\
0 & -1
\end{array}\right), \quad \sigma_{2}=\left(\begin{array}{cc}
0 & 1 \\
1 & 0
\end{array}\right), \quad \sigma_{3}=\left(\begin{array}{cc}
0 & -i \\
i & 0
\end{array}\right)
$$

denote the Pauli matrices in $M_{2}$. Then we can construct a spin system $\left\{s_{1}, \ldots, s_{2 n}\right\}$ in $M_{2^{n}}=\bigotimes_{1}{ }^{n} M_{2}$ as follows, where $1 \leq k<n-1$.

$$
\begin{aligned}
& s_{1}=\sigma_{1} \otimes 1^{\otimes n-1} \\
& s_{2}=\sigma_{2} \otimes 1^{\otimes n-1} \\
& \cdot \\
& \cdot \\
& s_{2 k+1}=\sigma_{3}^{\otimes k} \otimes \sigma_{1} \otimes 1^{\otimes n-k-1} \\
& s_{2 k+2}=\sigma_{3}^{\otimes k} \otimes \sigma_{2} \otimes 1^{\otimes n-k-1} \\
& \cdot \\
& \cdot \\
& s_{2 n-1}=\sigma_{3}^{\otimes n-1} \otimes \sigma_{1} \\
& s_{2 n}=\sigma_{3}^{\otimes n-1} \otimes \sigma_{2}
\end{aligned}
$$

where for $a \in M_{2}, a^{\otimes k}$ denotes the k-fold tensor product of $a$ with itself.

Let $V_{m}$ denote the linear span of $s_{0}=1, s_{1}, \ldots, s_{m}$. Then $V_{m}$ is a spin factor of dimension $\mathrm{m}+1$ in $M_{2^{n}}$. For $\mathrm{m}=2 \mathrm{n}$ the $C^{*}$-algebra $C^{*}\left(V_{m}\right)$ generated by $V_{2 n}$ equals $M_{2^{n}}$, so in that case $V_{m}$ is irreducible, see [3], Theorem 6.2.2. If $\mathrm{m}=2 \mathrm{n}-1$ then $C^{*}\left(V_{m}\right)=M_{2^{n-1}} \bigoplus M_{2^{n-1}} \subset M_{2^{n}}$.

By [1] or [8] , Proposition 2.2.10, if $\operatorname{Tr}$ denotes the usual trace on $M_{2^{n}}$ then there exists a positive idempotent map $P: M_{2^{n}} \rightarrow V_{m}+i V_{m}$ given by $\operatorname{Tr}(P(a) b)=\operatorname{Tr}(a b)$ for all $a \in M_{2^{n}}, b \in V_{m}+i V_{m}, m \leq 2 n$. Then $P$ restricted to the self-adjoint part of $M_{2^{n}}$ is a projection map onto $V_{m}$. With the HilbertSchmidt structure the set $\left\{1, s_{1}, \ldots, s_{m}\right\}$ is an orthonormal basis for $V_{m}$ with respect to the normalized trace $2^{-n} \operatorname{Tr}$ on $M_{2^{n}}$. Thus $P$ has the form

$$
P(a)=2^{-n} \sum_{0}^{m} \operatorname{Tr}\left(s_{i} a\right) s_{i} .
$$


By [7] or [8], Theorem 2.3.4P is atomic if $n \neq 2,3,5$. By [3], Theorem 6.2.3 $V_{m}$ is a JW-factor of type $I_{2}$, i.e. for each minimal projection $e \in V_{m}, 1-e$ is also a minimal projection. Thus $\operatorname{Tr}(e)=2^{n-1}$. Note that for each $e_{i}, i \geq 1, e_{+}=$ $1 / 2\left(1+s_{i}\right)$ and $e_{-}=1 / 2\left(1-s_{i}\right)$ are such projections.

Let $t$ denote the transpose on $M_{2}$. Then

$$
\sigma_{1}^{t}=\sigma_{1}, \quad \sigma_{2}^{t}=\sigma_{2}, \quad \sigma_{3}^{t}=-\sigma_{3} .
$$

Since the transpose on $M_{2^{n}}$ is the tensor product $t^{\otimes n}$, it follows from the defining equations for $s_{k}$ that

$$
s_{2 k+1}^{t}=(-1)^{k} s_{2 k+1}, \quad s_{2 k+2}^{t}=(-1)^{k} s_{2 k+2} .
$$

It follows in particular that $P \circ t=t \circ P$.

Lemma 8 Define a symmetry $W \in M_{2^{n}}$ as follows:

(i) If $n$ is odd, $n=2 m+1, W=\left(1 \otimes \sigma_{3}\right)^{\otimes m} \otimes 1$.

(ii) If $n$ is even, $n=2 m, W=\left(1 \otimes \sigma_{3}\right)^{\otimes m}$.

Then $A d W\left(s_{k}\right)=s_{k}^{t}$ for all $1 \leq k \leq 2 n$. Hence $A d W(a)=a^{t}$ for all $a \in V_{n}$.

Furthermore, if $n$ is of the form $n=4 m+i, i=0,1$, then $W \in C^{*}\left(V_{n}\right)$.

Proof. If $k=1,2$, then $A d W\left(s_{k}\right)=s_{k}=s_{k}{ }^{t}$, so we may assume $k \geq 3$. We first consider the case when $k=2 j+1$ with $\mathrm{j}$ odd. Then

$$
s_{k}=\sigma_{3}^{\otimes j} \otimes \sigma_{1} \otimes 1^{\otimes n-j-1} .
$$

Thus by definition of $W$, since $A d \sigma_{3}\left(\sigma_{1}\right)=-\sigma_{1}$, we have

$$
A d W\left(s_{k}\right)=\sigma_{3}^{\otimes j} \otimes\left(-\sigma_{1}\right) \otimes 1^{\otimes n-j-1}=-s_{k}=(-1)^{j} s_{k}=s_{k}^{t} .
$$

Similarly if $k=2 j+2$ with $\mathrm{j}$ odd, then $\operatorname{AdW}\left(s_{k}\right)=s_{k}{ }^{t}$. Now let $k=2 j+1$ with $\mathrm{j}$ even. Then

$$
A d W\left(s_{k}\right)=\sigma_{3}^{\otimes j} \otimes \sigma_{1} \otimes 1^{\otimes n-j-1}=s_{k}=(-1)^{j} s_{k}=s_{k}^{t} .
$$

Similarly for $k=2 j+2$ with $\mathrm{j}$ even. Thus in every case $A d W\left(s_{k}\right)=s_{k}^{t}$. Since $V_{n}$ is the real linear span of $s_{k}, \mathrm{k}=0,1, \ldots, \mathrm{n}, A d W(a)=a^{t}$ for all $a \in V_{n}$

If $n=4 m+i, i=0,1$, then, since $\sigma_{3}^{t}=-\sigma_{3}$, and there are $2 m$ factors of $\sigma_{3}$ in $W$, we have $W^{t}=W$. If $i=0$ then by [3], Theorem $6.2 .2, C^{*}\left(V_{n}\right)=M_{2^{n}}$, so clearly $W \in C^{*}\left(V_{n}\right)$. If $n=4 m+1$ then again by [3], Theorem 62.2

$$
C^{*}\left(V_{4 m+1}\right)=M_{2^{4 m}} \bigoplus M_{2^{4 m}} \subset M_{2^{4 m+1}} .
$$

Since in this case $W=\left(1 \otimes \sigma_{3}\right)^{\otimes 2 m} \otimes 1$, it follows that $W \in M_{4^{m}} \otimes \mathbb{C} \subset$ $C^{*}\left(V_{4 m+1}\right)=C^{*}\left(V_{n}\right)$, completing the proof of the lemma.

Lemma 9 Let $m \leq 2 n$ and $P: M_{2^{n}} \rightarrow V_{m}$ be the trace invariant projection. Let $W$ be as in Lemma \%. Then

$$
P=P \circ t \circ A d W .
$$


Proof. By Lemma 8 if $a \in V_{m}$ then $t \circ \operatorname{AdW}(a)=a$. Thus if $x \in M_{2^{n}}$ then

$$
(P \circ t \circ A d W) \circ(P \circ t \circ A d W)(x)=P \circ(P \circ t \circ A d W)(x)=P \circ t \circ A d W(x) .
$$

Thus $P \circ t \circ A d W$ is idempotent with range $V_{m}$ and being the identity on $V_{m}$. Since $P$ is trace invariant, if $x \in M_{2^{n}}, y \in V_{m}$ we have

$$
\begin{aligned}
\operatorname{Tr}(P \circ t \circ A d W(x) y)= & \operatorname{Tr}(t \circ A d W(x) y)=\operatorname{Tr}\left(A d W(x) y^{t}\right) \\
& =\operatorname{Tr}(x A d W \circ t(y))=\operatorname{Tr}(x y)=\operatorname{Tr}(P(x) y),
\end{aligned}
$$

using that $A d W \circ t=t \circ A d W=\iota$ on $V_{m}$, where $\iota$ is the identity map on $V_{m}$. The lemma follows.

The following lemma is probably well known, but is included for completeness.

Lemma 10 Let $a \in B(H)$ be positive and $e, f$ projections in $B(H)$ with sum 1. Then

$$
2(e a e+f a f) \geq a .
$$

Proof. We have

$$
a=(e+f) a(e+f)=e a e+e a f+f a e+f a f .
$$

Let

$$
b=(e-f) a(e-f)=e a e-e a f-f a e+f a f \geq 0 .
$$

Thus

$$
a \leq a+b=2(e a e+f a f),
$$

as asserted.

We shall need the following slight extension of a result of Robertson [6]. For simplicity we show it in the finite dimensional case. Recall that $M^{\prime}$ denotes the commutant for a set $M \subset B(H)$ and that $B_{s a}$ denotes the set of self-adjoint operators in $M$.

Lemma 11 Let $H$ be a finite dimensional Hilbert space, and let $B \subset B(H)$ be a $C^{*}$-algebra and $A \subset B_{\text {sa }}$ a Jordan algebra with $1 \in A$. Suppose $P: B_{s a} \rightarrow A$ is a positive projection map. Suppose $\phi \leq P$ is a completely positive map, $\phi: B \rightarrow B$. Then $\phi(1) \in C^{*}(A)^{\prime}$, and $\phi(x)=\phi(1) x$ for $x \in C^{*}(A)$.

Proof. By [8], Lemma 2.3.5, since $P(x)=x$ for $x \in A, \phi(1) \in A$, and $\phi(x)=$ $\phi(1) x=x \phi(1)$, for $x \in A$. Since $C^{*}(A)$ is the $C^{*}$-algebra generated by $A, \phi(1) \in$ $C^{*}(A)^{\prime}$. Since $H$ is finite dimensional, if $e$ is the range projection of $\phi(1), \phi(1)$ has a bounded inverse $\phi(1)^{-1}$ on $e H$. Thus

$$
\psi=\phi(1)^{-1} e \phi
$$

is a unital map of $B$ into $e B e$ such that for $x \in A$,

$$
\psi(x)=\phi(1)^{-1} e \phi(x)=\phi(1)^{-1} \phi(1) x=e x .
$$


Thus $\psi_{\mid A}$ is a Jordan homomorphism, so $A \subset D=\left\{x \in B_{s a}: \psi\left(x^{2}\right)=\psi(x)^{2}\right\}$, the definite set for $\psi$. Since $\psi$ is completely positive, by [6] or [8], Proposition 2.1.8, $D$ is the self-adjoint part of a $C^{*}$-algebra, hence $\psi$ is a homomorphism on $C^{*}(A)$. Since by the above $\psi(x)=e x$ for $x \in A, \psi(x)=e x$ for $x \in C^{*}(A)$. If $x \in C^{*}(A), 0 \leq x \leq 1$ then $\phi(x) \leq \phi(1)=e \phi(1)$. Thus $\phi(x)=e \phi(x)$, so that for all $x \in C^{*}(A)$, we have

$$
\phi(x)=e \phi(x)=\phi(1) \psi(x)=\phi(1) e x=\phi(1) x,
$$

proving the lemma.

Lemma 12 Let $P: M_{2^{n}} \rightarrow V_{m}, m \leq 2 n$ be the trace invariant projection Then $P \geq 2^{-n} \iota$, and $P \geq 2^{-n} t \circ A d W$, with $W$ as in Lemma 8. Furthermore there exists a 1-dimensional projection $q \in M_{2^{n}}$ such that $P(q)=2^{-n} 1$, hence

$$
2^{-n}=\max \{\lambda \geq 0: P \geq \lambda \iota\} .
$$

Proof. Let $p$ be a 1-dimensional projection in $M_{2^{n}}$. Since $V_{m}$ is a JW-factor of type $I_{2}, 3$, Theorem 6.1.8, there are two minimal projections $e$ and $f$ in $V_{m}$ with sum 1 and $a, b \geq 0$ such that

$$
P(p)=a e+b f .
$$

By [8, Proposition 2.1.7, $P($ epe $)=e P(p) e=a e$, so that

$$
a 2^{n-1}=\operatorname{Tr}(a e)=\operatorname{Tr}(P(\text { epe }))=\operatorname{Tr}(\text { epe }) .
$$

Hence

$$
a=2^{-n+1} \operatorname{Tr}(\text { epe }), b=2^{-n+1} \operatorname{Tr}(f p f) .
$$

Since epe is positive of rank $1, \operatorname{Tr}($ epe $) \geq$ epe. Thus, using Lemma 10 we get

$$
\begin{aligned}
P(p) & =2^{-n+1}(\operatorname{Tr}(\text { epe }) e+\operatorname{Tr}(f p f) f) \\
& \geq 2^{-n+1}(\text { epe }+f p f) \\
& \geq 2^{-n+1} \frac{1}{2}(\text { epe }+ \text { epf }+f p e+f p f) \\
& =2^{-n} p .
\end{aligned}
$$

Since this holds for all 1-dimensional projections $p, P \geq 2^{-n} \iota$. By Lemma 9 it thus follows that

$$
P=P \circ t \circ A d W \geq 2^{-n} t \circ A d W,
$$

proving the first part of the lemma.

To show the second part we exhibit a 1-dimensional projection $q$ such that $P(q)=2^{-n} 1$. The Pauli matrix $\sigma_{3}$ is of the form $\sigma_{3}=e_{0}-f_{0} \in M_{2}$ with $e_{0}, f_{0}$ 1-dimensional projections in $M_{2}$. Let $T r_{2}$ denote the usual trace on $M_{2}$. Then for $j=1,2$, we have

$$
\begin{aligned}
0 & =\operatorname{Tr}_{2}\left(\sigma_{3} \sigma_{j}\right)=\operatorname{Tr}_{2}\left(e_{0} \sigma_{j}\right)-\operatorname{Tr}_{2}\left(f_{0} \sigma_{j}\right) \\
& =\operatorname{Tr}_{2}\left(e_{0} \sigma_{j}-\left(1-e_{0}\right) \sigma_{j}\right) \\
& =2 \operatorname{Tr}_{2}\left(e_{0} \sigma_{j}\right)-\operatorname{Tr}_{2}\left(\sigma_{j}\right) \\
& =2 \operatorname{Tr}_{2}\left(e_{0} \sigma_{j}\right) .
\end{aligned}
$$


Furthermore, $\operatorname{Tr}_{2}\left(e_{0} \sigma_{3}\right)=\operatorname{Tr}_{2}\left(e_{0}\left(e_{0}-f_{0}\right)\right)=\operatorname{Tr}_{2}\left(e_{0}\right)=1$. Let $q=e_{0}^{\otimes n} \in M_{2^{n}}$. If $j=2 k-i, i=0,1$, then $s_{j}=\sigma_{3}^{\otimes k-1} \otimes \sigma_{j} \otimes 1^{\otimes n-k}$. From the above we thus have

$$
\operatorname{Tr}\left(q s_{j}\right)=\operatorname{Tr}_{2}\left(e_{0} \sigma_{j}\right)=0 .
$$

Thus, since $s_{0}=1$, we have

$$
P(q)=2^{-n}\left(\sum_{0}^{m} \operatorname{Tr}\left(q s_{j}\right) s_{j}\right)=2^{-n} \operatorname{Tr}\left(q s_{0}\right) s_{0}=2^{-n} 1,
$$

completing the proof.

The projection $q$ above is not symmetric because $\sigma_{3}^{t}=-\sigma_{3}=f_{0}-e_{0}$, so that $e_{0}^{t}=f_{0}$. Furthermore $\operatorname{AdW}(q)=A d W\left(e_{0}^{\otimes n}\right)=q$, hence $t \circ \operatorname{AdW}(q)=q^{t} \perp q$. These properties of q will limit our choice of $V_{m}$ in our study of $P$.

In the case $m=2^{n}$ there are four classes of non-isomorphic irreducible Jordan subalgebras of $\left(M_{m}\right)_{s a}$, namely $\left(M_{m}\right)_{s a}$ itself, $V_{2 n}, S_{m}$, the real symmetric matrices in $M_{m}$, and $M_{2^{n-1}}(\mathbb{H})_{s a}$, the self-adjoint $2^{n-1} \times 2^{n-1}$ matrices over the quaternions $\mathbb{H}$ represented as $2 \times 2$ matrices, see [3], Ch. 6. Presently we shall specialize to the case when $V_{2 n} \subset\left(M_{2^{n-1}}\right)_{s a}$. We refer the reader to [4] for further information on this case.

With our previous notation with $W$ defined as in Lemma 8 let

$$
Q(X)=\frac{1}{2}(x+t \circ A d W(x)) .
$$

Then $Q$ is the projection of $M_{2^{n}}$ onto the fixed point set of the anti-automorphism $t \circ A d W$, hence by Lemma 8 is the projection onto the reversible Jordan algebra $A_{2 n}$ containing $V_{2 n}$. Thus, if $V_{2 n} \subset M_{2^{n-1}}(\mathbb{H})_{s a}$ then $Q: M_{2^{n}} \rightarrow M_{2^{n-1}}(\mathbb{H})_{s a}$.

Lemma 13 With the above notation, if $V_{2 n} \subset A_{2 n}=M_{2^{n-1}}(\mathbb{H})_{\text {sa }}$ and $P$ the projection $P: M_{2^{n}} \rightarrow V_{2 n}$, then

$$
P=\left.P\right|_{A_{2 n}} \circ Q \geq 2^{-n+1} Q .
$$

Proof. It suffices to show $P(p) \geq 2^{-n+1} p$ for all minimal projections $p$ in $A_{2 n}$. For such a projection $\operatorname{Tr}(p)=2$. We have $P(p)=a e+b f, a, b \geq 0$, as in the proof of Lemma 12. Then $a=2^{-n+1} \operatorname{Tr}($ epe $), b=2^{-n+1} \operatorname{Tr}(f p f)$. Since $p$ is a minimal projection in $A_{2 n}$, pep $=\lambda p, p f p=\mu p$ with $\lambda, \mu \geq 0$. Then

$$
(\text { epe })^{2}=\text { epepe }=\lambda \text { epe } .
$$

Since rank epe $=$ rank $p e p=2$, epe $=\lambda_{0} e_{0}$ with $e_{0}$ a projection in $A_{2 n}$ of dimension 2. Thus

$$
\lambda_{0} 2=\operatorname{Tr}\left(\lambda_{0} e_{0}\right)=\operatorname{Tr}(\text { epe })=\operatorname{Tr}(\text { pep })=\operatorname{Tr}(\lambda p)=\lambda 2 .
$$


Therefore $\lambda_{0}=\lambda$. Thus epe $=\lambda e_{0}$, and similarly $f p f=\mu f_{0}$. We thus have, since $e \geq e_{0}$ and $f \geq f_{0}$,

$$
\begin{aligned}
P(p) & =2^{-n+1}(\operatorname{Tr}(\text { epe }) e+\operatorname{Tr}(f p f) f) \\
& =2^{-n+1}\left(\operatorname{Tr}\left(\lambda e_{0}\right) e+\operatorname{Tr}\left(\mu f_{0}\right) f\right) \\
& \left(2 \lambda e_{0}+2 \mu f_{0}\right) \\
& =2^{-n+1}(2 e p e+2 f p f) \\
\geq & 2^{-n+1}(\text { epe }+e p f+f p e+f p f) \\
& =2^{-n+1} p
\end{aligned}
$$

where we used Lemma 10. The proof is complete.

Lemma 14 Given $V_{2 n}$ and $A_{2 n}$ as above, and assume $A_{2 n} \cong M_{2^{n-1}}(\mathbb{H})_{s a}$. Then there exists a 1-dimensional projection $q$ in $M_{2^{n}}$ such that $Q(q)=\frac{1}{2}\left(q+q^{t}\right)$ with $q \perp q^{t}, P(q)=2^{-n} 1$, and $\beta=P-2^{-n+1} Q$ is bi-optimal.

Proof. By Lemma $\left.13 P\right|_{A_{2 n}} \geq 2^{-n+1} \iota$. Since $P=P \circ Q$ we therefore have $\beta=P \circ Q-2^{-n+1} Q \geq 0 . V_{2 n}$ is irreducible by [3], Theorem 6.2.2, so $C^{*}\left(V_{2 n}\right)=$ $M_{2^{n}}$, so by Lemma 12 there is a 1-dimensional $q \in C^{*}\left(V_{2 n}\right)$ such that $2^{-n} 1=$ $P(q)=P(Q(q))$. By the comments after Lemma 12, $q^{t}=t \circ A d W(q) \perp q$, so in particular

$$
Q(q)=\frac{1}{2}(q+\circ A d W(q))=\frac{1}{2}\left(q+q^{t}\right) .
$$

Furthermore

$$
\beta(Q(q))=P(Q(q))-2^{-n+1} Q(q)=2^{-n}\left(1-\left(q+q^{t}\right)\right) .
$$

To show $\beta$ is bi-optimal, let $\phi \leq \beta$ be completely positive. Then by Lemma 11, $\phi(x)=\phi(1) x=\lambda x, \lambda \geq 0$, since $\phi(1) \in C^{*}\left(V_{2 n}\right)^{\prime}=\mathbb{C}$. Thus

$$
\lambda\left(q+q^{t}\right)=\phi\left(q+q^{t}\right)=2 \phi(Q(q)) \leq 2 \beta(Q(q))=2^{-n}\left(1-\left(q+q^{t}\right)\right) .
$$

Since $q+q^{t} \perp 1-\left(q+q^{t}\right), \lambda=0$, so $\phi=0$. Thus $\beta$ is optimal.

Next, if $\phi \leq \beta$ is copositive, then $t \circ \phi$ is completely positive, and

$$
t \circ \phi \leq t \circ P=P \circ t=P \circ A d W,
$$

since $P=P \circ t \circ A d W$ by Lemma 9 . Thus by Lemma $11 t \circ \phi=\lambda \iota$ with $\lambda \geq 0$. Hence

$\lambda\left(q+q^{t}\right)=\operatorname{to} \phi\left(q+q^{t}\right)=2 \operatorname{to} \phi(Q(q)) \leq 2 t \circ \beta(Q(q))=2^{-n}\left(1-\left(q+q^{t}\right)\right)^{t}=2^{-n}\left(1-\left(q+q^{t}\right)\right)$,

so again $\lambda=0$, and $\phi=0$. Thus $\beta$ is bi-optimal, completing the proof to the lemma.

From the above we see that if $\phi \leq P$ is completely positive or copositive, then $\phi \leq \lambda Q$ for some $\lambda \geq 0$. Since $P \geq \alpha=2^{-n+1} Q$, and $P(q)=2^{-n} 1$, it follows that $\alpha$ is a maximal decomposable map majorized by $P$.

Summarizing Lemma 14 and the above comments we obtain the following result. 
Theorem 15 Assume the reversible Jordan algebra $A_{2 n}$ containing $V_{2 n}$ is isomorphic to $M_{2^{n-1}}(\mathbb{H})_{s a}$, and let $Q: M_{2^{n}} \rightarrow A_{2 n}$ be the trace invariant projection. Let $\alpha=2^{-n+1} Q$ and $\beta=P-\alpha$. Then $P=\alpha+\beta$ is a decomposition as in Theorem 5 .

The following result describes Theorem 7 in detail for $P$.

Theorem 16 Let $P: M_{2^{n}} \rightarrow V_{2 n}$ be the trace invariant projection. Let $\alpha=$ $2^{-n} \iota$, and $\beta=P-2^{-n} \iota$, where $\iota$ is the identity map. Then $\alpha$ is a maximal completely positive map majorized by $P, \beta$ is optimal, and $P=\alpha+\beta$.

Proof. By Lemma $12 P \geq \alpha$, so $\beta \geq 0$, and there exists a 1-dimensional projection $q \in M_{2^{n}}$ such that $P(q)=2^{-n} 1$. Since $V_{2 n}$ is irreducible the argument in the proof of Lemma 14 shows that if $\phi \leq \beta$ is completely positive, then $\phi=\lambda \iota$ with $\lambda \geq 0$. Thus

$$
\lambda q=\phi(q) \leq \beta(q)=2^{-n} 1-2^{-n} q=2^{-n}(1-q),
$$

which implies $\lambda=0$. Thus $\beta$ is optimal. As remarked before the statement of Theorem $15 \alpha$ is a maximal completely positive map majorized by $P$. The proof is complete.

It was crucial in the proof of Theorem 15 that $A_{2 n}=M_{2^{n-1}}(\mathbb{H})_{s a}$, so $\operatorname{dimq}=$ 2 for a minimal projection $q$ in $A_{2 n}$. In the case when $A_{2 n}=S_{2^{n}}$, the real $2^{n} \times 2^{n}$ matrices, we have been unable to find a 1-dimensional projection $p \in A_{2 n}$ such that $P(p)=2^{-n} 1$, so that for each minimal projection $e \in V_{2 n}$ we have

$$
\operatorname{Tr}(p e)=\operatorname{Tr}(\text { epe })=\operatorname{Tr}(P(\text { epe }))=\operatorname{Tr}(e P(p) e)=\operatorname{Tr}\left(e 2^{-n} 1\right)=\frac{1}{2},
$$

so $\operatorname{Tr}(p$.$) is the trace on V_{2 n}$.

If $n=1, V_{2}=S_{2}=A_{1}$, so $\operatorname{Tr}(p$. $)$ is never a trace on $A_{1}$. We next show this for $V_{4}$ too, showing in particular the well known result that $A_{2}=M_{2}(\mathbb{H})_{s a}$. We thus leave it as an open question whether there is $\mathrm{n}$ such that $\operatorname{Tr}(p$.) can be a trace on $V_{2 n}$ for a 1-dimensional projection $p \in A_{2 n}$, or even for $p \in M_{2^{n}}$.

Example 17 . If $n=2$ then there is no positive rank 1 operator $x \in M_{4}$ such that $t \circ A d W(x)=x$.

Proof. Let $\bar{\phi}: M_{2} \rightarrow M_{2}$ be defined by

$$
\bar{\phi}\left(\begin{array}{ll}
a & b \\
c & d
\end{array}\right)=\left(\begin{array}{cc}
d & -c \\
-b & a
\end{array}\right)
$$

Then $\bar{\phi}=A d \sigma_{3}$ as is easily seen. Let $\phi=t \circ \bar{\phi}$. Then $\phi$ is an anti-automorphism of order 2 , and

$$
\phi\left(\begin{array}{ll}
a & b \\
c & d
\end{array}\right)=\left(\begin{array}{cc}
d & -b \\
-c & a
\end{array}\right)
$$


is such that $\mathcal{R}=\left\{A \in M_{2}: \phi\left(A^{*}\right)=A\right\}$ is the quaternions. Also $\phi=t \circ \sigma_{3}$. For simplicity of notation let $\rho=A d \sigma_{3}$. Let $T$ denote the $4 \times 4$ matrix

$$
\left(\begin{array}{cc}
A & B \\
C & D
\end{array}\right)
$$

with $A, B, C, D \in M_{2}$. Then

$$
\iota \otimes \rho\left(T^{*}\right)=\left(\begin{array}{cc}
\rho(A)^{*} & \rho(C)^{*} \\
\rho(B)^{*} & \rho(D)^{*}
\end{array}\right)
$$

Therefore

$$
t \circ(\iota \otimes \rho)\left(T^{*}\right)=\left(\begin{array}{cc}
t \circ \rho(A)^{*} & t \circ \rho(B)^{*} \\
t \circ \rho(C)^{*} & t \circ \rho(D)^{*}
\end{array}\right)
$$

Thus $t \circ(\iota \otimes \rho)\left(T^{*}\right)=T$ if and only if

$$
A=\phi\left(A^{*}\right), B=\phi\left(B^{*}\right), C=\rho\left(C^{*}\right), D=\phi\left(D^{*}\right)
$$

if and only if $A, B, C, D \in \mathbb{H}$, and so $T \in M_{2}(\mathbb{H})$. But $M_{2}(\mathbb{H})$ contains no positive rank 1 operators, so there is no positive rank $1 x \in M_{4}$ such that $t \circ A d W(x)=x$, completing the proof of the example.

If $\mathcal{P}=\left\{s_{i}: i \in \mathbb{N}\right\}$ is an infinite spin system then the norm closed linear span $V_{\infty}$ of 1 and $\mathcal{P}$ is the infinite spin factor. The $C^{*}$-algebra $C^{*}\left(V_{\infty}\right)$ generated by $V_{\infty}$ is the CAR-algebra $A$ which is isomorphic to the infinite tensor product of $M_{2}$ with itself, see e.g. [3], Theorem 6.2.2. By [1], Lemma 2.3, there exist a unique trace invariant positive projection $P$ of $C^{*}\left(V_{\infty}\right)_{s a}$ onto $V_{\infty}$. If $M_{2^{n}}=$ $\otimes_{1}^{n} M_{2}$ is imbedded in $C^{*}\left(V_{\infty}\right)$ by $x \rightarrow x \otimes 1 \in M_{2^{n}}^{\infty} \otimes \otimes_{n+1}^{\infty} M_{2}$, it is clear that $\left.P\right|_{M_{2^{n}}}=P_{n}$, the trace invariant projection onto $V_{2 n}$. Thus if $\phi \leq P$ is decomposable then $\left.\phi\right|_{M_{2^{n}}} \leq\left. P\right|_{M_{2^{n}}}=P_{n}$ for $\mathrm{n}$ even. Thus by Lemmas11 and $12,\left.\phi\right|_{M_{2^{n}}} \leq\left. 2^{-n} \iota\right|_{M_{2^{n}}}$. But if $m \geq n$ is even then

$$
\left.\phi\right|_{M_{2^{n}}}=\left.\left(\left.\phi\right|_{M_{2^{m}}}\right)\right|_{M_{2^{n}}} \leq\left. 2^{-m}\left(\left.\iota\right|_{M_{2^{m}}}\right)\right|_{M_{2^{n}}} .
$$

Thus

$$
\left.\phi\right|_{M_{2^{n}}} \leq\left. 2^{-m} \iota\right|_{M_{2^{n}}}
$$

for all even $m \geq n$. Thus $\phi=0$. Similarly if $\phi \leq t \leq P$. We have thus shown

Corollary 18 Let $P$ be the projection of the self-adjoint part of the CARalgebra onto the spin factor $V_{\infty}$. Then $P$ is bi-optimal.

\section{References}

[1] E.Effros and E. Størmer,Positive projections and Jordan structure in operator algebras, Math. Scand. 45(1) (1979), 127-138.

[2] K-C. Ha and S-H. Kye, Optimality for indecomposable entanglement witnesses, Physical Review , 86 (2012), 034301. 
[3] H. Hanche-Olsen and E. Størmer, Jordan operator algebras, Monographs and Studies in Mathematics, vol 21 (Pitman Advanced Publishing Program), Boston 1984.

[4] H.B. Lawson and M-L. Michelson, Spin geometry, Princeton University Press, 1981.

[5] M. Marciniak, On extremal positive maps acting between type I factors, Noncommutative Harmonic Analysis with Applications to probability II, Banach Center Publications, vol. 89 (2010), 201-221.

[6] A.G. Robertson, Positive extensions of automorphisms of spin factors, Proc. Royal Society of Edinburgh, 94A, (1983), 71-77.

[7] A.G. Robertson, Positive projections on $C^{*}$-algebras and an extremal map, J. London Math. Soc. (2) 32 (1), (1985), 133-140.

[8] E. Størmer, Positive linear maps of operator algebras, Springer Monographs in Math. (2013), Springer-Verlag.

Department of Mathematics, University of Oslo, 0316 Oslo, Norway.

e-mail erlings@math.uio.no 